\title{
Beitrag zum Vorkommen und Ausbreitung des Trauer-Rosenkäfers Oxythyrea funesta (PoDA, 1761) (Coleoptera, Scarabaeidae) in Deutschland: ein Citizen-Science-Projekt
}

\author{
Mit 7 Figuren und 1 Tabelle
}

Hannes Hoffmann ${ }^{1}$, Fabian A. Boetzl ${ }^{2}$, Gernot Medger ${ }^{3}$, Daniel Rolke ${ }^{4}$ und Christoph Benisch ${ }^{5}$

1 Christian-Albrechts-Universität zu Kiel, Institut für Natur- und Ressourcenschutz, Abteilung für Landschaftsökologie, Olshausenstrasse 75, 24118 Kiel, Deutschland. - hanneshoffmann89@gmx.de

2 Lehrstuhl für Tierökologie und Tropenbiologie, Biozentrum, Am Hubland, 97074 Würzburg. - fabian.boetzl@uni-wuerzburg.de

3 Bailleuler Straße 3, 59368 Werne. - gernot.medger@t-online.de

${ }^{4}$ Landesamt für Umweltschutz Sachsen-Anhalt, Reideburger Straße 47, 06116 Halle (Saale). - daniel.rolke@lau.mlu.sachsen-anhalt.de

5 Windmühlstraße 21,68165 Mannheim. - cbenisch2@kerbtier.de

Published on 2021-06-30

DOI:10.21248/contrib.entomol.71.1.137-146

\section{Zusammenfassung}

Der auffällig gezeichnete Trauerrosenkäfer Oxythyrea funesta konnte sich in den letzten 30 Jahren in Mitteleuropa rasant ausbreiten und eignet sich auch deshalb als Modell für Citizen-Science-Projekte. Bei „kerbtier.de“ wurde 2019 ein Kartierungsprojekt gestartet, um die Art durch Fotos der Nutzer gezielt zu melden und die Ausbreitung in Deutschland besser zu dokumentieren. Es wurden insgesamt 1.345 Anfragen von 309 Nutzern ausgewertet. Unsere Ergebnisse bestätigen, dass Oxythyrea funesta mittlerweile aus allen Regionen Deutschlands nachgewiesen ist und in der südlichen Hälfte Deutschlands eine weit verbreitete und häufige Art ist. In Norddeutschland liegt ihr aktueller Verbreitungsschwerpunkt in und um die Städte Hannover, Bremen und Hamburg. Generell scheint die Art vom Klimawandel zu profitieren, sowie durch das Vorhandensein von ausreichend Blütenpflanzen und Komposthaufen in urbanen Gebieten, wo die Art überwiegend dokumentiert wurde. Die Eignung von Citizen-Science-Projekten in der Biodiversitätsforschung wird diskutiert.

\section{Schlüsselwörter}

Coleoptera, Scarabaeidae, Blatthornkäfer, Cetoniinae, Rosenkäfer, Klimawandel, Faunistik, Paläarktis, Bürgerwissenschaft, Mitteleuropa

\section{Abstract}

The characteristic white-spotted rose beetle Oxythyrea funesta has spread rapidly in Central Europe over the past 30 years and is therefore a suitable model for Citizen Science projects. A project to document the dispersal of O. funesta in Germany was started on "kerbtier.de" in 2019 to record the species through photos uploaded by the users. 1,345 inquiries from 309 users were evaluated. Our results show that $O$. funesta is currently present in all regions of Germany and became a widespread and common species in the southern half of Germany. In Northern Germany, its current distribution is focused in and around the cities of Hanover, Bremen and Hamburg. In general, the species 
appears to benefit from climate change, as well as from the presence of sufficient flowering plants and compost heaps in urban areas where the species has been mainly documented. The suitability of Citizen-Science-projects in biodiversity research is discussed.

\section{Key words}

Coleoptera, Scarabaeidae, scarab beetle, Cetoniinae, flower chafer, climate change, faunistics, Palearctic, Citizen Science, Central Europe

\section{Einleitung}

Der Trauer-Rosenkäfer Oxythyrea funesta (PoDA, 1761) ist glänzend schwarz gefärbt mit weißen Flecken auf der Ober- und Unterseite, und weist kurz nach der Imaginalhäutung eine Behaarung auf, welche mit zunehmendem Alter verloren geht (Fig. 1). Dabei erreicht er eine Größe von acht bis zwölf Millimetern. Durch dieses markante Äußere ist $O$. funesta einfach und auch durch Laien im Feld anzusprechen und in der heimischen Fauna höchstens mit Tropinota hirta (PoDA, 1761) zu verwechseln.

O. funesta besitzt einen einjährigen Entwicklungszyklus. Die Larven entwickeln sich in acht bis zehn Wochen vor allem in substratreichem, organischem Material pflanzlicher Herkunft wie Kompost- oder Misthaufen (Mico \& Galante 2003, Horák et al. 2013). Die adulten Käfer ernähren sich von Blüten vor allem niedrigwüchsiger Pflanzen aus den Familien der Asteraceae, Apiaceae, Rosaceae, Brassicaceae und Fabaceae (Rössner 2012, TAMutis \& Dapkus 2013, Thomaes et al. 2016). Dabei gilt die Art in Südeuropa durchaus als Schädling, da die Käfer in größerer Anzahl Knospen, Blüten und Früchte schädigen können (Vuts et al. 2008, SuBChEv et al. 2012).

O. funesta besitzt ein großes Verbreitungsgebiet in der gesamten westlichen Paläarktis von Nordafrika im Süden, Großbritannien im Westen und Südfinnland im Norden bis nach Zentral-Sibirien im Osten (SMETANA 2006, HorÁк 2016). Dabei gilt die Art als pontisch-mediterranes Faunenelement, das ausgehend von den Urstromtälern der Donau über Main und Rhein nach Mitteleuropa eingewandert ist (Horion 1958). LoRENZ (1996) diskutiert außerdem eine Ausbreitung über das Elbtal durch angeschwemmtes Totholz. Bis in die Mitte der 1990er Jahre war O. funesta nur selten und wenn, dann in Wärmegebieten zu finden. Seitdem hat die Art ihr Verbreitungsgebiet in den mitteleuropäischen Ländern rasant erweitert, was von vielen Autoren beobachtet und beschrieben wurde (z. B. Tamutis \& Dapkus 2013, Barclay \& Notton 2015, Thomaes et al. 2016).

In Deutschland ist O. funesta seit 1994 wieder regelmäßig bei Aschaffenburg anzutreffen. Seitdem konnten zahlreiche Wiederfunde und Neumeldungen aus den Bundesländern erbracht werden. In Sachsen gibt es erste Nachweise seit 1994 und in den Folgejahren konnte sich die Art hier landesweit ausbreiten (z. B. LoRENZ 1996, ZiNke 1997, Dietrich \& BRÄUER 2010, Rössner 2012, Klausnitzer et al. 2018). Im Jahr 1995 erreichte O. funesta Nordhessen westlich von Kassel (Schaffrath 1997).
Aus Westfalen gibt es seit 1999 Nachweise (Drees 1999, Diener 2012, Medger 2019). Ab 2002 gelangen regelmäßige Nachweise für den Bayerischen Wald, auch in Höhenlagen von über 700 Metern (BussLer 2007). Für Thüringen liegen, neben sehr alten Meldungen (RössNER 2012), ein unsicherer Nachweis aus dem Jahr 2001 und sichere aktuelle Nachweise ab 2010 vor (BÄsE 2016). Aus der Region Nordrhein existieren Funde wieder seit 2006 (Niehuis \& Weitzel 2009, Junker \& Köhler 2010, HÖRREN 2011) und aus der Region Hannover seit 2011 (von Holdt 2012). Schleswig-Holstein und Hamburg erreichte die Art 2012 (GüRLICH et al. 2018). In SachsenAnhalt gibt es Nachweise seit 2013 (Neumann et al. 2014). Für das Weser-Ems-Gebiet konnte der Erstnachweis 2014 erbracht werden (BELLMANN et al. 2018). Im Jahr 2015 gelangen die Erstnachweise aus dem südlichen Brandenburg (BÄSE 2016, EssER 2016), nach zuvor vermutlich verschleppten Tieren in Berlin (HENDRICH 2005). Folglich fehlten aktuelle Nachweise nur noch für die Region des Niederelbegebietes und Mecklenburg-Vorpommern.

Aufgrund ihrer Auffälligkeit, der aktuell bereits weiten Verbreitung und der leichten Auffindbarkeit als Blütenbesucher eignet sich O. funesta sehr gut als Modell für Citizen-Science-Ansätze (Bürgerwissenschaft). Citizen Science ist ein vor allem in der Biodiversitätsforschung wachsender Wissenschaftszweig, bei welchem die Datenerhebung vorrangig durch interessierte Laien durchgeführt wird. Diese Daten können durch Wissenschaftlerinnen und Wissenschaftler ausgewertet und verwendet werden. Dabei fördern diese Projekte das Schließen von Wissenslücken der Teilnehmer, sowie deren Einstellung und Verhalten in Bezug zur Biodiversität (PEter et al. 2019). Natürlich beschränkt sich das Konzept von Citizen Science hierbei auf die Erfassung gut kenntlicher Arten und kann alleine nicht zur Erfassung und Bewertung der heimischen Flora und Fauna dienen. Dennoch gibt es z. B. mit dem „Tagfalter-Monitoring Deutschland“ (KüHN et al. 2019) oder den Projekten „Die Stunde der Gartenvögel“ und „Insektensommer“ vom NABU zahleiche positive Beispiele von CitizenScience-Projekten in Deutschland.

Um Daten von „citizen scientists“ zentral sammeln und verwerten zu können, braucht es geeignete Datenbanken, vorzugsweise online. Mit seiner Fotogalerie von über 2.600 der etwa 7.000 Arten der Käferfauna Deutschlands bietet „kerbtier.de“ Hilfe beim 
Bestimmen von Käfern. Dafür essentiell ist das Bestimmungsforum. Nutzer können hier mitteleuropäische Käfer anhand ihrer Fotos zusammen mit Angaben zu Größe, Fundort und Funddatum einreichen und vom „kerbtier.de“-Bestimmungsteam identifizieren lassen. Bei erfolgreichen Bestimmungen auf Artniveau werden diese Meldungen dann für Verbreitungskarten und Phänogramme weiter verwendet, sodass jeder Nutzer zur ständigen Erweiterung der Datengrundlage beiträgt. Außerdem können Nutzer Arten nach zahlreichen Kriterien suchen und Fotos mehrerer Arten miteinander vergleichen. In der Sektion Fauna finden sich Nachweise bemerkenswerter Käferarten sowie Module zur Anzeige ihrer geographischen Verbreitung und ihres jahreszeitlichen Auftretens.

Um die schnelle Ausbreitung von O. funesta in Deutschland zu dokumentieren, wurde zu Beginn des Jahres 2019 auf „kerbtier.de“ ein Aufruf zur gezielten Kartierung dieser Art gestartet. Dabei wurden alle interessierten Nutzer aufgefordert, verstärkt auf diese Art zu achten und aktuelle und zurückliegende Nachweise mit Hilfe von Fotos zu melden sowie deren Fundumstände (z. B. Habitat, Blütenpflanze) beizufügen, um folgende Fragestellungen zu bearbeiten:

(1) Wie weit konnte sich O. funesta bis Ende 2019 in Deutschland ausbreiten?

(2) In welchen Habitaten kommt O. funesta am häufigsten vor und an welchen Pflanzen kann sie dort bevorzugt angetroffen werden?

(3) Wie groß ist das Potenzial von Citizen Science für die Kartierung und Datengewinnung von gut kenntlichen Arten in einem Biodiversitätsprojekt?

\section{Material und Methoden}

In der Auswertung wurden alle Meldungen von O. funesta auf „kerbtier.de“ bis Ende des Jahres 2019 berücksichtigt. Anfragen ohne präzise Datumsangabe wurden nicht verwendet. Die Fundorte mussten in Deutschland liegen bzw. in einem Messtischblatt mit deutschem Anteil. Für die Auswertung wurden anhand der Fotos und mit Hilfe des Anfragetextes der Nutzer folgende Informationen, soweit möglich, gewonnen: Bundesland, faunistische Käferregion, Messtischblatt, Funddatum, Familie/Gattung/Art/Blütenfarbe der Fundpflanze und der Habitattyp des Fundortes. Bei mehreren Anfragen desselben Nutzers am gleichen Tag die in allen Kategorien identisch waren, wurde nur eine Anfrage gewertet, um den Einfluss von Nutzern mit sehr vielen Anfragen so gering wie möglich zu halten. Die Blütenfarben von rosa über rot bis lila wurden dabei zu einer Kategorie („rot“) zusammengefasst. Die Pflanzenart wurde oft nur bis auf Gattungsniveau bestimmt und ging dann auch als diese in die Auswertung ein. Die Fundorte der Art wurden je nach Beschreibung der Nutzer zu Habitattyp-Kategorien zusammengefasst (Tab. 1).

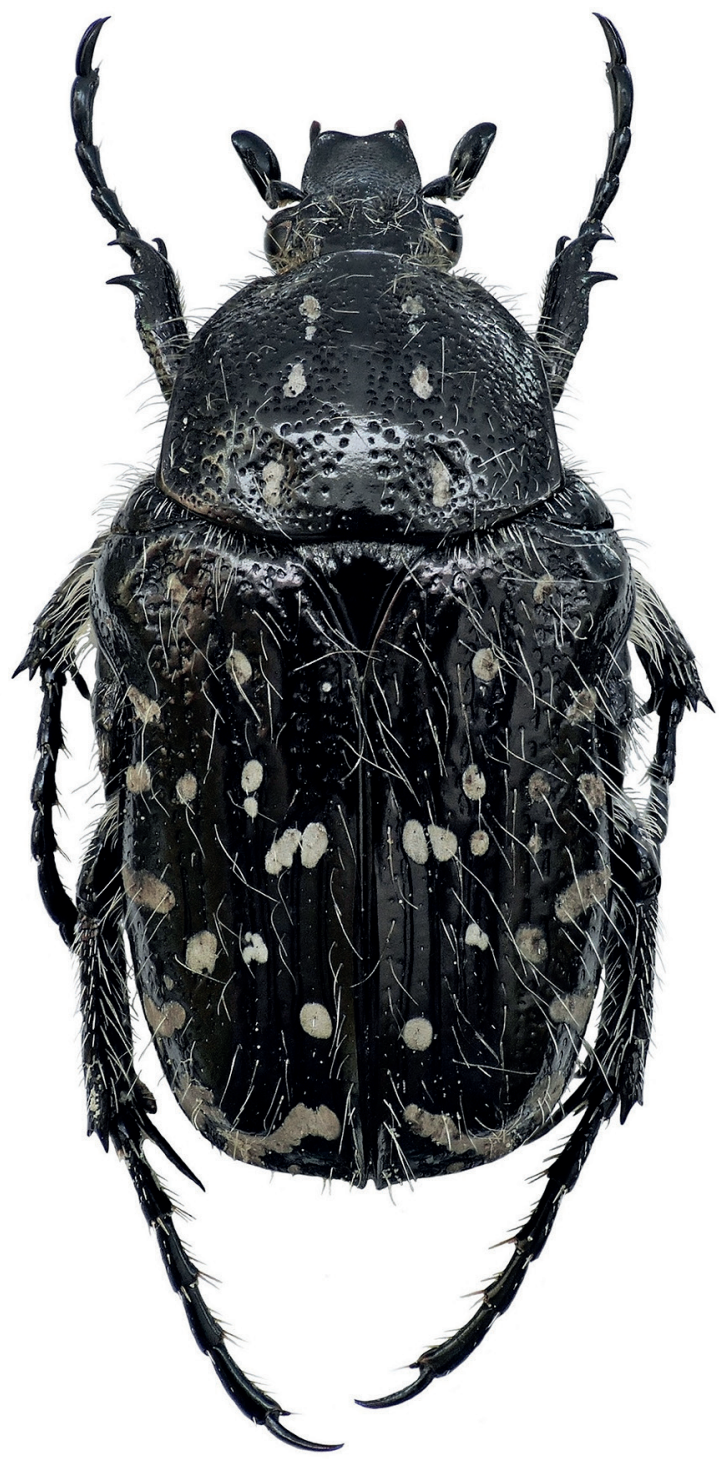

Fig. 1: Habitusvon Oxythyreafunesta(PoDA, 1761). - Aufnahme: G. Medger.

\section{Ergebnisse}

\section{Verbreitung und Phänologie}

Insgesamt gingen 1.345 Fotoanfragen von 309 Nutzern mit Funden aus den Jahren 2005 bis 2019 ein (Fig. 2), die für die weitere Auswertung genutzt wurden. Dabei reichte jeder Nutzer im Durchschnitt 3,9 Anfragen ein.

O. funesta konnte während des Kartierungsprojektes in 440 TK 25 Messtischblättern verteilt über alle Bundesländer und mit Ausnahme des Niederelbegebietes auch für alle faunistischen Käferregionen nachgewiesen werden (Fig. 3). Die meisten Meldungen stammten aus den Käferregionen Baden, Sachsen, Hessen und Nordbayern (Fig. 4). Dabei gelang mit einer Meldung vom Plauer See im Landkreis „Mecklenburger Seenplatte“ aus dem Jahr 2018 auch der Erstnachweis der Art für Mecklenburg-Vorpommern. Eine weitere Meldung lag für ein Messtischblatt mit Anteilen der Regionen 
„Niederelbegebiet“ und „Weser-Ems-Gebiet“ vor. Da genaue Koordinaten dieses Fundortes fehlen, konnte diese Meldung leider keiner Region sicher zugeordnet werden. Die Messtischblätter „8414: Laufenburg (Baden)“ mit 5,2\%, gefolgt von „5118: Marburg“ mit $3,7 \%$ und „5035: Jena“ mit 3,5\% waren jene mit den meisten Anfragen.

O. funesta konnte von Mitte März bis Ende September beobachtet werden und erreichte ihr Aktivitätsmaximum von Mitte Mai bis Ende Juli (Fig. 5).

Tab. 1: Legende zur Auswahl der Habitattyp-Kategorien.

\begin{tabular}{|l|l|}
\hline Habitat & Fundorte \\
\hline Gärten & Gärten, Parks, Friedhöfe \\
Wiesen & $\begin{array}{l}\text { aller Art } \\
\text { aller Art }\end{array}$ \\
Säume & $\begin{array}{l}\text { Ufer, Moore, Auen, Gräben } \\
\text { Weuchtgebiete }\end{array}$ \\
Sërmegebiete & $\begin{array}{l}\text { Binnendünen, Weinberge, Mager- } \\
\text { rasen, Heidegebiete } \\
\text { Truppenübungsplätze, Tongruben, } \\
\text { Tagebau, Talsperren, Steinbrüche, } \\
\text { Stromtrassen, Kiesgruben, Sand- } \\
\text { gruben, Golfplätze, Baggerseen, } \\
\text { Deponien, Bahndämme } \\
\text { Brachen, Ruderalflächen } \\
\text { Streuobstwiesen } \\
\text { Brachen }\end{array}$ \\
$\begin{array}{l}\text { Streuobstwiesen } \\
\text { Gehölzstrukturen } \\
\text { Blühstreifen }\end{array}$ & \begin{tabular}{l} 
aller Art Hecken, Gehölze \\
\hline
\end{tabular} \\
\hline
\end{tabular}

\section{Fundpflanzen und Habitate}

O. funesta konnte auf 41 Pflanzenfamilien nachgewiesen werden. Am häufigsten besuchte sie Arten der Asteraceae (47\%), Apiaceae (14\%), Rosaceae (10\%), Caprifoliaceae (6\%), Ranunculaceae (4\%), Fabaceae (3\%) und Brassicaceae (2\%; Fig. 6a). 15 Pflanzenfamilien wurden nur einmal besucht.

Dabei besuchte O.funesta 120 Pflanzenarten. Am häufigsten flog sie Leucanthemum spp. (13\%), Centaurea spp. (12\%), Cirsium spp. (11\%), Rosa spp. (6 \%), Knautia arvensis (6\%), Taraxacum spp. (5\%), Ranunculus spp. (4\%), Achillea millefolium (4\%) und Heracleum sphondylium (4\%) an (Fig. 6b).

O. funesta wurde zu $45 \%$ auf weißen, gefolgt von $35 \%$ auf rosa und $18 \%$ auf gelben Blüten nachgewiesen ( $\mathrm{n}=1.176$; Fig. 6c). Nur 22-mal (2\%) flog sie blaue Blüten an.

O. funesta wurde weiterhin $\mathrm{zu} 32 \%$ in Gärten, $19 \%$ auf Wiesen, $14 \%$ in Säumen, $7 \%$ in Feuchtgebieten, $7 \%$ in Wärmegebieten, $6 \%$ in Sekundärhabitaten, $5 \%$ in Brachen, $5 \%$ in Streuobstwiesen, $3 \%$ in Gehölzstrukturen und $\mathrm{zu} 2 \%$ in Blühstreifen nachgewiesen (Fig. 6d).

\section{Diskussion}

\section{Verbreitung}

O. funesta hat ihr Verbreitungsgebiet in den letzten Jahren rasant ausgedehnt und ist aktuell bis Mitteldeutschland zur Linie Dortmund-Göttingen-Halle-Cottbus sehr häufig anzutreffen. Lediglich an der südlichen Grenze zwischen Baden-Württemberg und Bayern gibt es noch eine größere Verbreitungslücke, die wohl auf wenige Nutzer und Meldungen aus dieser Region zurückzuführen ist. In den nördlichen Landesteilen liegt ihr Hauptvorkommen in den Großstädten Bremen, Hannover und Hamburg sowie deren Umland. Hier konnte sich die Art nach dem ersten Auftreten früh etablieren und in alle Richtungen ausbreiten. Die Funde aus Berlin werden weiterhin als verschleppte Tiere angesehen (siehe auch HeNDRICH 2005). Der Erstnachweis aus Mecklenburg-Vorpommern geht wahrscheinlich ebenfalls auf ein verschlepptes Tier zurück, da die nächstgelegensten Nachweise weit entfernt liegen (ca. $150 \mathrm{~km}$ ). Die Entwicklung der dortigen Population bleibt abzuwarten. Dennoch kann davon ausgegangen werden, dass sich die Art auch in Norddeutschland, wie schon in den meisten Gebieten Süddeutschlands, weiter ausbreiten wird.

\section{Besondere Beobachtungen von Nutzern}

\section{Entwicklung}

Im Nachfolgenden möchten wir auf besondere Beobachtungen der „kerbtier.de“-Nutzer eingehen. Mit Hilfe der Anfrage-ID lassen sich diese Meldungen zu jeder Zeit auf „kerbtier.de“ nachvollziehen.

Anfrage 125.175: „Ich habe die Käfer in einem Sack mit gesiebtem Kompost gefunden. Im Kompost (ausschließlich Grünschnitt und Holz aus dem Garten) habe ich im letzten Jahr Cetonia aurata und Protaetia cuprea gefunden. Es scheint nunmehr so, dass winzige Larven oder Eier von Oxythyrea funesta im Gesiebe verblieben sind und es im Sack bis zur [sic!] Imago geschafft haben. Die Entwicklung findet also wie bei anderen Cetoninae, zumindest bei mir, nicht im Rasen o. ä. statt, sondern in verrottendem Substrat."

Anfrage 125.422: "Schlupffrisch in der Komposterde eines Beetes."

Anfrage 159.294: „....in einer Tonne mit Kompost gefunden. Das Kompostiergut war sechs Wochen vorher umgesetzt worden. Dabei tauchten an die 100 Engerlinge von mindestens zwei Arten in unterschiedlicher Größe auf. Sie wurden mit der grob gesiebten Erde in der Tonne "gesammelt". Garten in Ortsrandlage."

Die Larven von O. funesta entwickeln sich vor allem in substratreichem, organischem Material pflanzlicher Herkunft wie Kompost-, Mist- oder Dunghaufen (Mico 


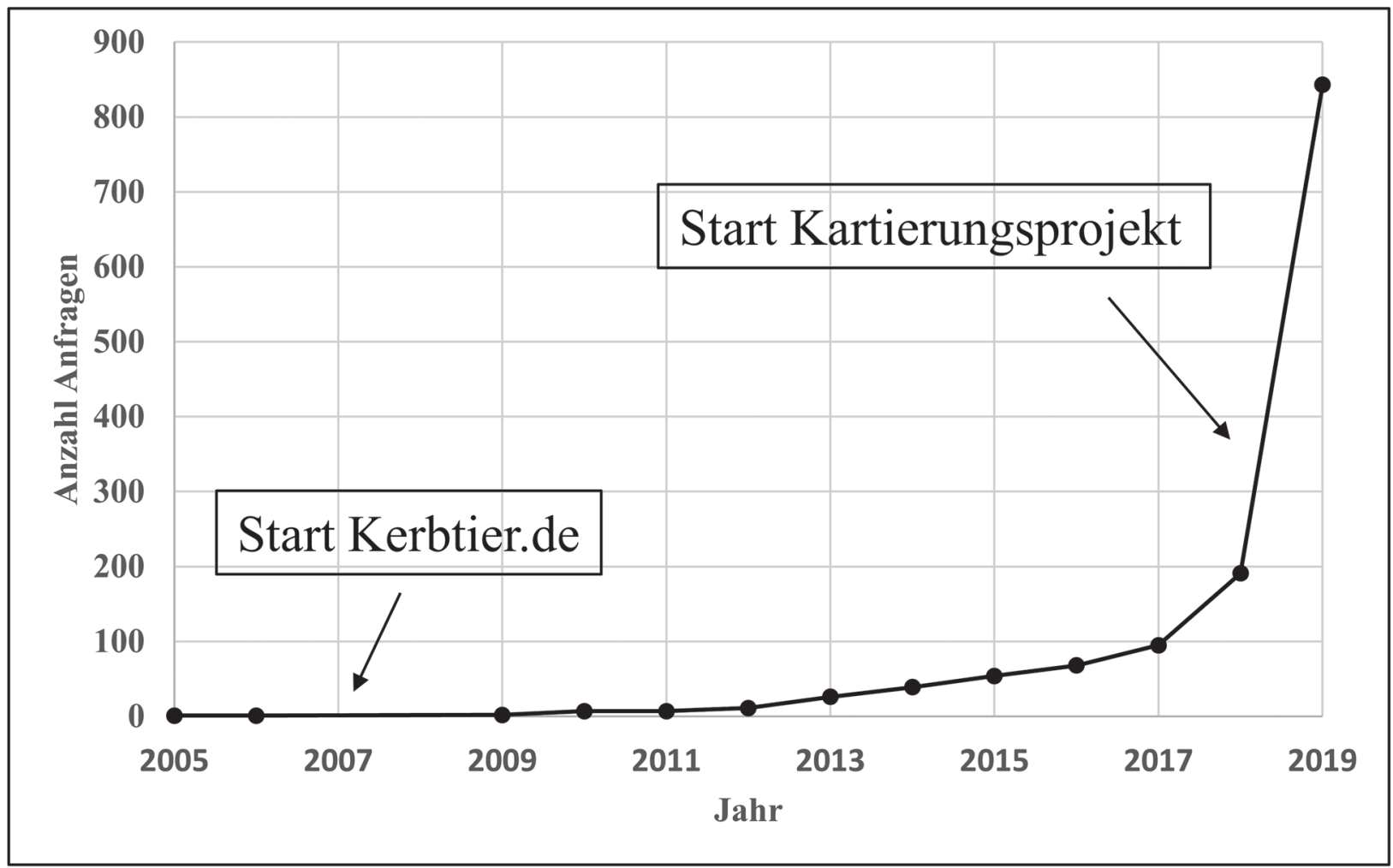

Fig. 2: Anzahl aller Anfragen von Oxythyrea funesta (PoDA, 1761) bei „kerbtier.de“ in den Jahren 2005 bis 2019.

\& Galante 2003, Horák et al. 2013). So gibt es z. B. Meldungen in Dunghaufen von Kaninchen (Mico \& Galante 2003) und aus einem Pferdemisthaufen, der auf einem Feld lagerte (Rössner 2012). Diese Erkenntnisse stimmen mit den Beobachtungen der "kerbtier.de“Nutzer überein. So scheint O. funesta in Komposthaufen ein geeignetes Entwicklungshabitat für die Larven $\mathrm{zu}$ finden. Die Komposthaufen könnten also eine entscheidende Rolle als Refugium und Trittstein für die weitere Ausbreitung spielen (ØDEgAARD \& TøMmeras 2000).

\section{Massenauftreten}

Anfrage 93.072: „Ein Massenbestand südlich und nördlich des Klingelborn von ca. 100 Oxythyrea funesta."

Anfrage 95.610: „Massenhaft! Ich will nicht übertreiben, aber im dreistelligen Bereich waren sie sicher vertreten." Anfrage 126.223: „Gefunden am Kanaldamm, bei 100 habe ich aufgehört zu zählen, hauptsächlich an Löwenzahn, aber auch an Apfel, Weißdorn und Traubenkirsche." Anfrage 140.553: „Handgezählte 49 Oxythyrea funesta auf Brombeerblüten in alter Kiesgrube.“

Einige Nutzer schildern Massenauftreten von O. funesta von bis zu über 100 Individuen auf teilweise eng begrenzten Flächen (Fig. 7). Die Art scheint sich nicht nur schnell auszubreiten, sondern sich unter günstigen Voraussetzungen auch schnell $\mathrm{zu}$ etablieren, fortzupflanzen und große Populationen aufzubauen. Solche Massenauftreten sind auch aus Ost- und Südeuropa bekannt, wo die Art dann durchaus schädlich an Kulturpflanzen auftreten kann (Vuts et al. 2008, Subchev et al. 2012).

\section{Fundpflanzen}

O. funesta wurde durch die Nutzer von „kerbtier.de“ zu fast $75 \%$ auf großen, weißen oder roten Blüten der Familien Asteraceae, Apiaceae und Rosaceae nachgewiesen, was mit den Erkenntnissen anderer Autoren übereinstimmt (z. B. Rössner 2012, Tamutis \& Dapkus 2013, Thomaes et al. 2016). Medvedev (1964) konnte sogar über 30 Pflanzenfamilien feststellen, die angeflogen wurden. Diese Anzahl liegt bei uns mit 41 nachgewiesenen Familien nochmals höher. Allerdings gab es bei 15 Pflanzenfamilien nur Einzelbeobachtungen. Es handelt sich hierbei um zumeist nicht-heimische Pflanzen in Parks und Gärten (z. B. Essigbaum Rhus typhina, Feigenkaktus Cylindropuntia imbricata, Rhododendron Rhododendron sp.). Generell konnte O. funesta aber je nach Jahreszeit zu etwa $75 \%$ auf nur zehn Pflanzengattungen nachgewiesen werden. Im April und Mai war sie beispielsweise zumeist auf Taraxacum spp. und Leucanthemum spp. zu finden, im Juni und Juli auf Rosa spp. und Knautia arvensis und im Juli und August auf Cirsium spp. und Tanacetum vulgare. O. funesta ist in der Blütenwahl demnach sehr generalistisch. Die Art nutzt die am häufigsten verfügbaren Pflanzen zur jeweiligen Jahreszeit und kann auch auf nicht-heimische und weniger häufige Pflanzenarten umsteigen, was einen wesentlichen Vorteil zur Etablierung und Ausbreitung der Art darstellt. 


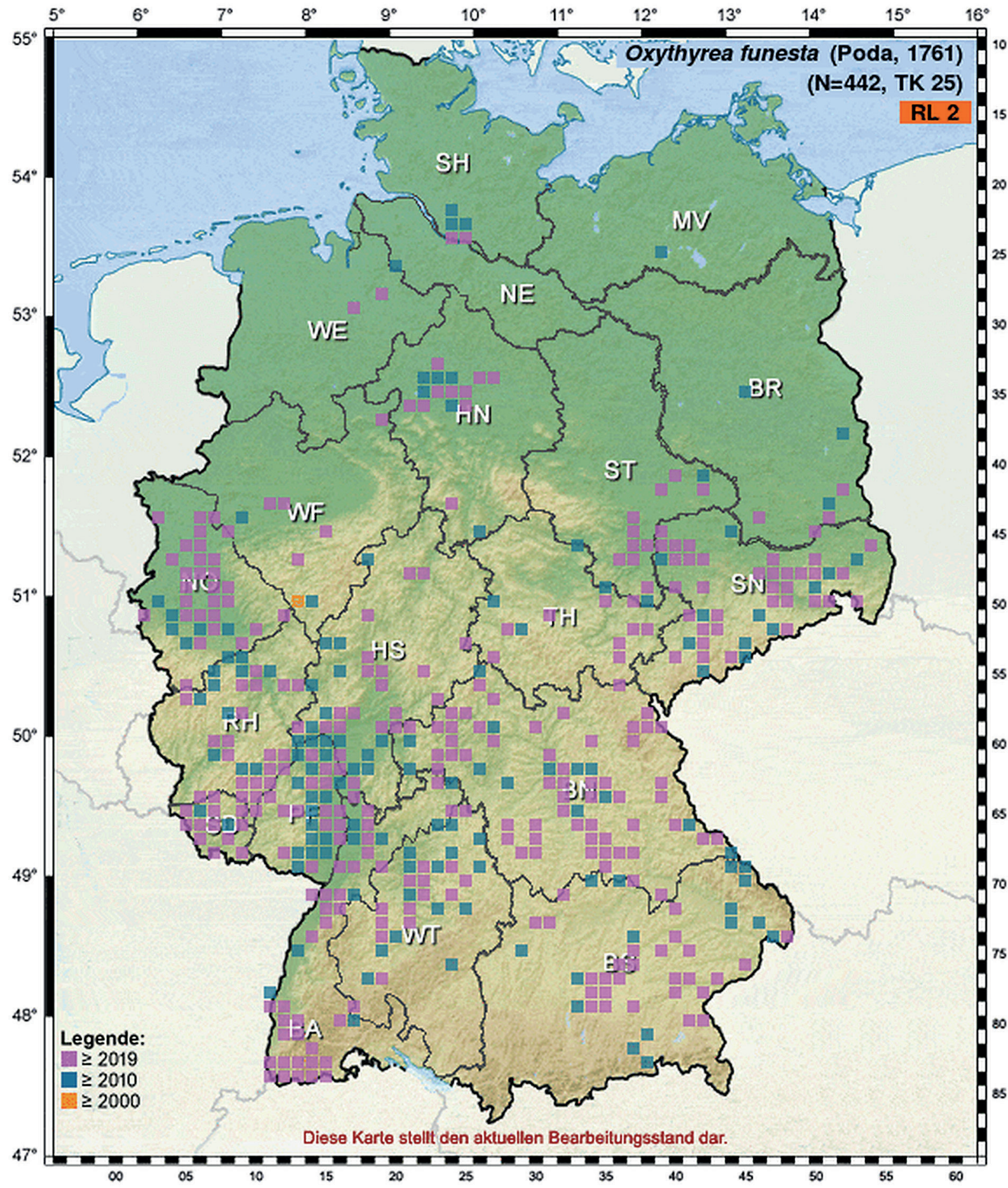

Fig. 3: Verbreitungskarte von Oxythyrea funesta (PoDA, 1761) in Deutschland basierend auf über 1.000 Fotoanfragen von über 300 Nutzern von „kerbtier.de“.

\section{Habitate}

O. funesta scheint nicht nur ihr Spektrum an Nahrungspflanzen ausgeweitet zu haben, sondern auch die Habitate in denen sie nun bevorzugt anzutreffen ist. Bis Mitte der 1990er Jahre war sie nur in Wärmegebieten und dort z. B. auf Magerrasen und in Weinbergen zu finden (Horion 1958). Unsere Ergebnisse zeigen eine starke Präferenz für Gärten und Parks (32\%), frische Wiesen
(19\%) und Saumstrukturen (14\%). Nur zu $7 \%$ wurde die Art aus Wärmegebieten gemeldet. Besonders nördlich der Mittelgebirge wurde die Art fast ausschließlich in Gärten und anderen Sekundärhabitaten gefunden. Dort scheint die Art vom reichen Angebot an Pflanzen und der Verfügbarkeit von Komposthaufen zu profitieren. Allerdings spiegeln die Meldungen auf „kerbtier.de“ 


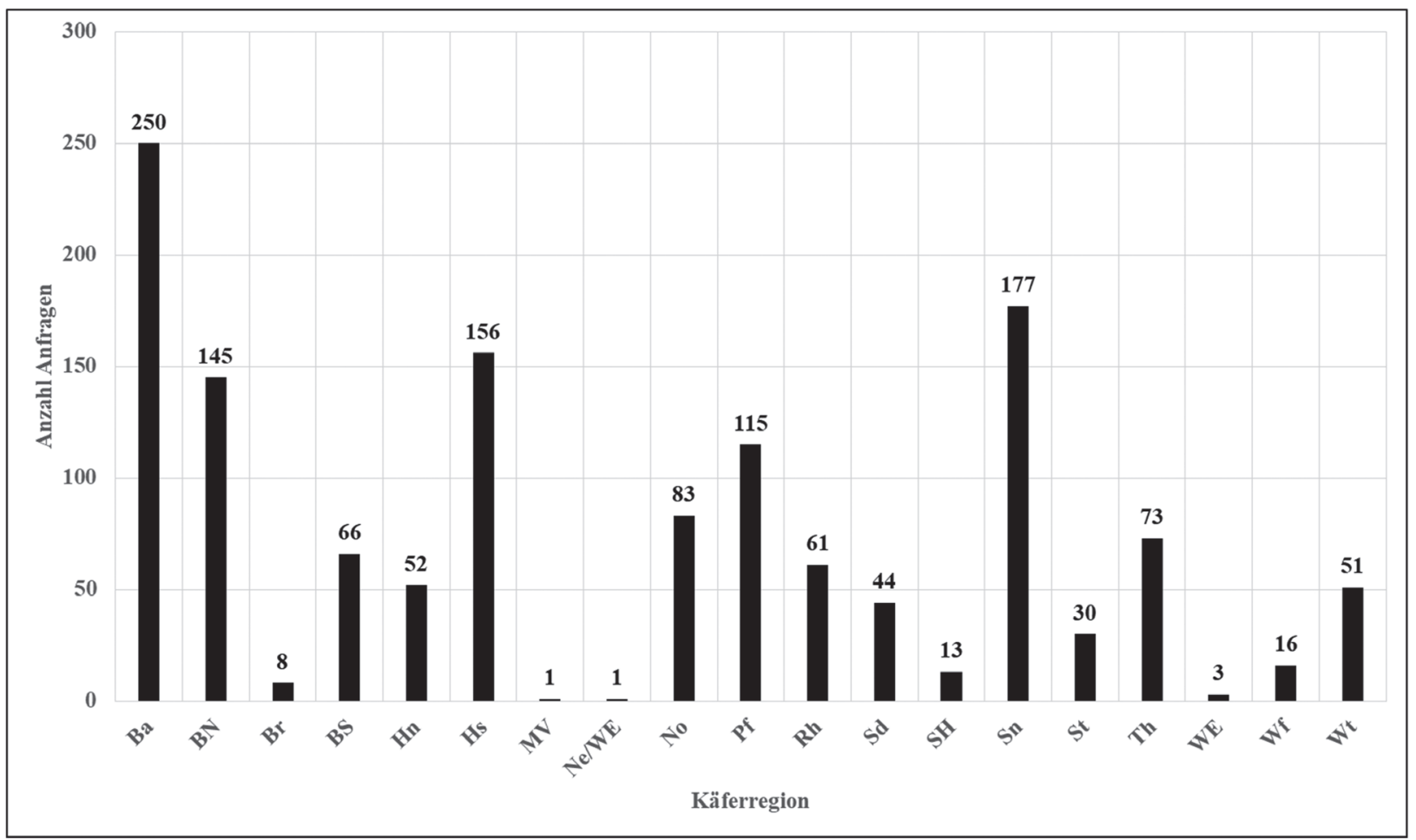

Fig. 4: Anzahl aller Anfragen von Oxythyrea funesta (PoDA, 1761) je Käferregion (Ba: Baden, BN: Bayern Nord, Br: Brandenburg, BS: Bayern Süd, Hn: Hannover, MV: Mecklenburg-Vorpommern, NE: Niederelbegebiet, No: Nordrhein, Pf: Pfalz, Rh: Rheinland, Sd: Saarland, SH: Schleswig-Holstein, Sn: Sachsen, St: Sachsen-Anhalt, Th: Thüringen, WE: Weser-Ems-Gebiet, Wf: Westfalen, Wt: Württemberg).

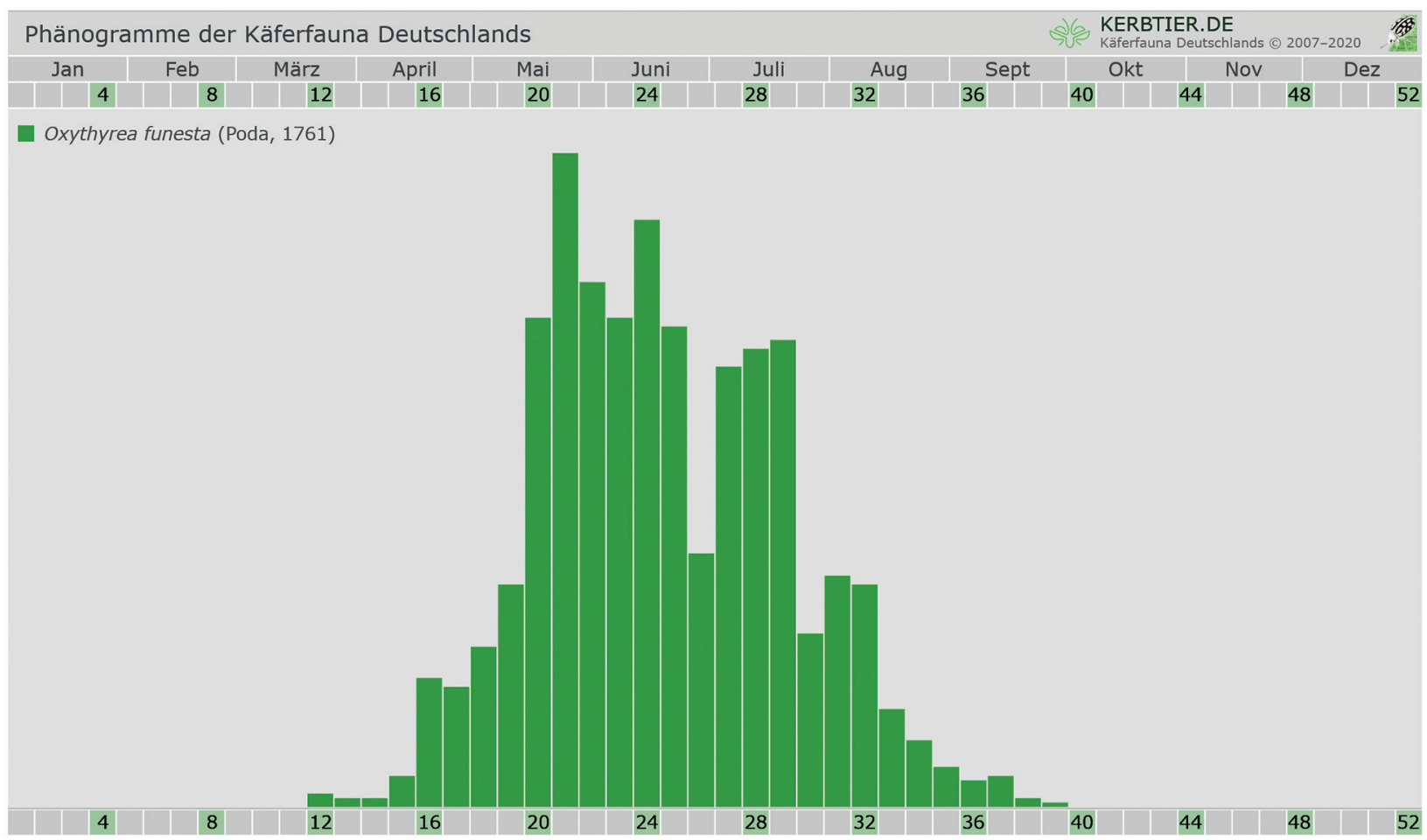

Fig. 5: Phänologie von Oxythyrea funesta (PoDA, 1761) basierend auf über 1.000 Fotoanfragen von über 300 Nutzern von „kerbtier.de“.

natürlich auch die Bewegungspräferenzen der Nutzer wider. Diese halten sich mutmaßlich vermehrt in ihren Gärten und in nahegelegenen Parks, sowie in Städte und Ortschaften umgebenden Landschaftsstrukturen wie extensiven Agrarflächen oder Randstrukturen auf. Wärmehabitate könnten dadurch untererfasst sein. Insgesamt zeigen unsere Ergebnisse allerdings deutlich, dass sich $O$. funesta auch abseits der wärmebegünstigten Habitate, aus denen sie bis in die 1990er Jahre bekannt war, ausbreiten konnte. 


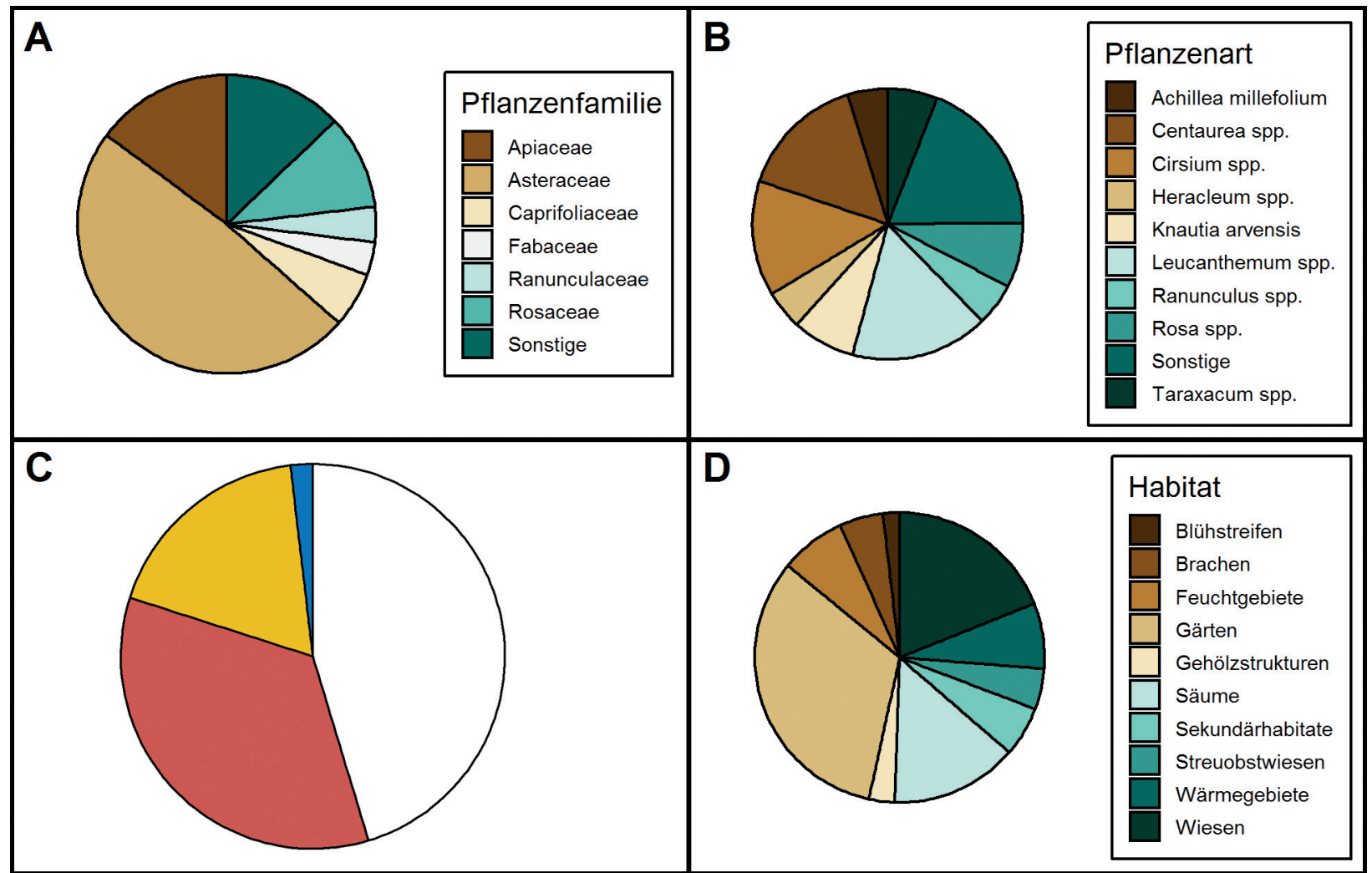

Fig. 6: Prozentuale Verteilung der häufigsten besuchten Pflanzenfamilien (A), Pflanzenarten (B), Blütenfarben (C) und Habitate (D) von Oxythyrea funesta (PoDA, 1761).

\section{Schlussfolgerung}

O. funesta ist bis zu den Mittelgebirgen eine häufige Art, die ihr Verbreitungsgebiet in den nächsten Jahren voraussichtlich weiter erweitern und dann auch in Norddeutschland regelmäßig bis häufig anzutreffen sein sollte. Es wird weiterhin vermutet, dass die Art durch Verschleppung mit z. B. Erd- oder Kompostmaterial in norddeutsche Großstädte wie Bremen, Hamburg und Hannover gelangt ist und sich dort etablieren und weiter ausbreiten konnte. Diese erfolgreiche und rasante Ausbreitung wird wohl durch den Klimawandel begünstigt, der wärmere Wintertemperaturen zur Folge hat, wovon vor allem die Larven profitieren. Des Weiteren ist die Art nicht auf bestimmte Pflanzen angewiesen, sondern ernährt sich von den zur jeweiligen Jahreszeit am häufigsten verfügbaren Pflanzen. Zusätzlich profitiert sie vom reichen Angebot an Pflanzen und der Verfügbarkeit von Komposthaufen in Gärten, Parks, Blühflächen und weiteren Sekundärhabitaten.

Um weitere Veränderungen in der heimischen Biodiversität registrieren und gegebenenfalls mit den Instrumenten des Artenschutzes tätig werden $\mathrm{zu}$ können, ist die kontinuierliche Erfassung von Beobachtungsdaten von entscheidender Wichtigkeit. Citizen Science kann hierfür unter bestimmten Voraussetzungen ein Baustein sein, um möglichst umfangreiche Daten zu bestimmten Arten zu erhalten (Pocock et al. 2018).
Die Dokumentation der Ausbreitung von O. funesta in Deutschland mit Hilfe von „kerbtier.de“ zeigt unter anderem das Potenzial von einer Plausibilitätskontrolle unterzogener Citizen Science im Bereich der Faunistik, und reiht sich somit in verschiedene Publikationen zu diesem Thema ein (MüNCH et al. 2010, KüHN et al. 2019).

Die gesamte heimische Artenvielfalt allein auf Grundlage von Citizen Science zu dokumentieren, erscheint jedoch unmöglich. So kann die verstärkte Integration von Citizen Science in Forschung und Artenschutz auch die Förderung klassischer taxonomischer und ökologischer Expertise nicht ersetzen.

Es sollte jedoch auch auf die Chance hingewiesen werden, die Expertise von interessierten Laien durch deren erfolgreiche Integration in Biodiversitätsprojekte zu vergrößern. Dies ist durchaus bei langjährigen Nutzern von „kerbtier.de“ zu beobachten. In einzelnen Fällen kann so zusätzliches Interesse geweckt und gefördert werden. So kann Citizen Science auch als Teil der Umweltbildung verstanden werden. Eventuell befassen sich manche Teilnehmer in der Folge verstärkt mit Fragen der Taxonomie, Ökologie und Faunistik und entwickeln sich somit über die Zeit zu (dringend benötigten) Artengruppenspezialisten. 


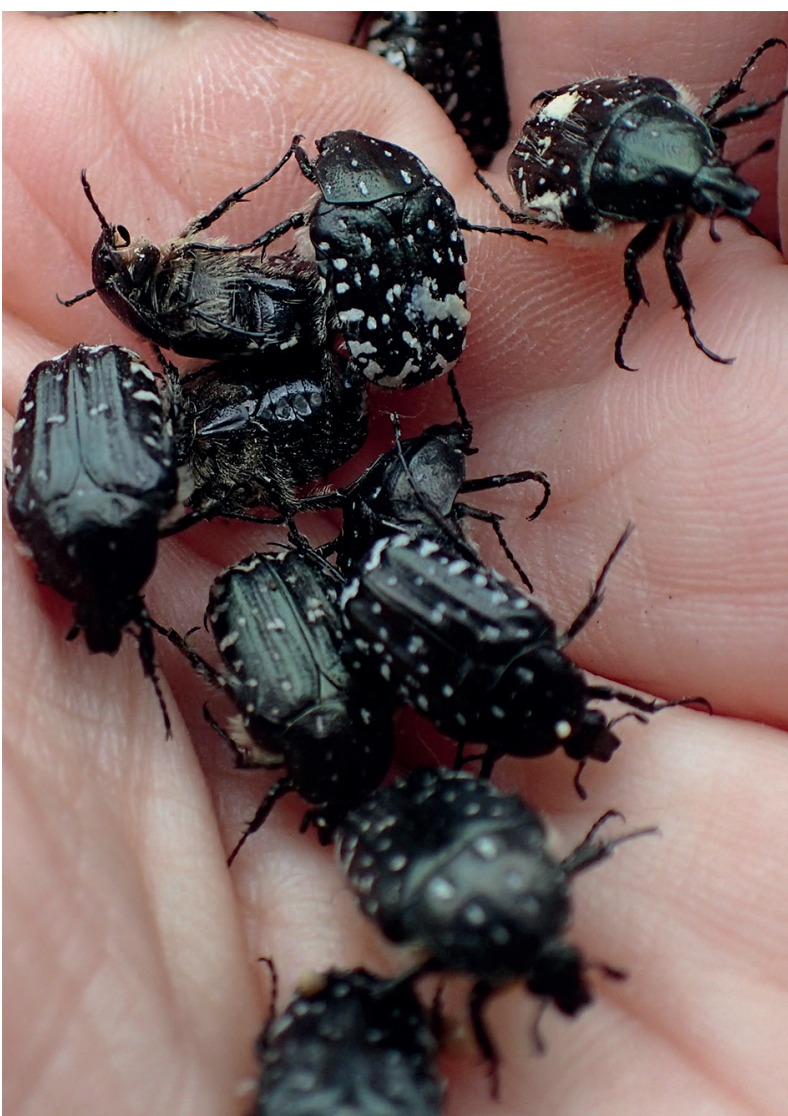

Fig. 7: Massenauftreten von Oxythyrea funesta (PoDA, 1761) in einer Heide im MTB „Euskirchen“ (Nordrhein). Aufnahme: C. Reetz, 14.07.2019.

\section{Danksagung}

Wir möchten uns ganz herzlich bei allen Nutzern bedanken, die seit dem Bestehen von „kerbtier.de“ mit ihren Anfragen zum Gelingen beigetragen haben und ohne die dieses Kartierungsprojekt nicht realisierbar gewesen wäre. Ein weiteres großes Dankeschön geht an unsere Teammitglieder von „kerbtier.de“ die durch ihre Bestimmungsarbeit ebenfalls einen großen Beitrag geleistet haben. Weiterhin möchten wir uns bei Stephan Gürlich (Buchholz) und Ludger Schmidt (Neustadt) für die Bereitstellung von Literatur und Informationen $\mathrm{zu}$ Funden aus Norddeutschland bedanken.

\section{Literaturverzeichnis}

Barclay, M. V. L. \& Notton, D. G. 2015: Oxythyrea funesta (PODA) (Scarabaeidae) in London and a summary of recent and historical British records of this species. - The Coleopterist 24 (2): 113-116.

Bäse, W. 2016: Oxythyrea funesta (PoDA, 1761) - Nachweis für Brandenburg (Coleoptera, Scarabaeidae). - Märkische Entomologische Nachrichten 18: $131-132$.
Bellmann, A.; Esser, J.; Janssen, R.; Mühlfeit, M. \& Reimann, S. 2018: Bemerkenswerte und neue Käferfunde aus dem Weser-Ems-Gebiet (Coleoptera) (Teil 12). - Abhandlungen des Naturwissenschaftlichen Vereins zu Bremen 47: 683-688.

Bussler, H. 2007: Wärmeliebende Rosenkäfer im Bayerischen Wald. - LWF aktuell 57: 58.

Diener, U. 2012: Neue Funde von Oxythyrea funesta (PODA) in Westfalen (Scarabaeidae). - Natur und Heimat 72: 91-92.

Dietrich, W. \& BRÄUER, S. 2010: Nachweise von Oxythyrea funesta im Erzgebirge/ Krusne hory (Coleoptera, Scarabaeidae). - Entomologische Nachrichten und Berichte 54: 258-259.

Drees, M. 1999: Neuer Fund von Oxythyrea funesta (PoDA) in Westfalen (Scarabaeidae). - Entomologische Blätter für Biologie und Systematik der Käfer 95: 165 .

Esser, J. 2016: Achter Nachtrag zum Verzeichnis der Käfer (Coleoptera) Brandenburgs und Berlins. - Märkische Entomologische Nachrichten 18: 109-115

GÜRlich, S.; Meybohm, H. \& Ziegler, W. 2018: Nachträge zur Käferfauna von Schleswig-Holstein, Hamburg und Nord-Niedersachsen. - Bericht der koleopterologischen Sektion mitzusammenfassendem Jahresrückblick 2012. - Bombus 4 (1): 14-25.

HENDRICH, L. 2005: Verschleppung von Oxythyrea funesta (PoDA, 1761) mit Schnittblumen/Topfpflanzen (Col., Scarabaeidae) nach Berlin. - Entomologische Nachrichten und Berichte 49: 150-151.

Horák, J.; Hui, C.; Roura-Pascual, N. \& Romportl, D. 2013: Changing roles of propagule, climate and land use during extralimital colonization of a rose chafer beetle. - Naturwissenschaften 100: 327-336. - doi: 10.1007/s00114-013-1029-2.

HoRÁk, J. 2016: Threatened or harmful? Opportunism across spatial scales apparently leads to success during extralimital colonisation. - Insect Conservation and Diversity 9: 351-357. - doi: 10.1111/icad.12174.

Horion, A. 1958: Faunistik der mitteleuropäischen Käfer. Band 6: Lamellicornia (Scarabaeidae, Lucanidae). August Feyel Verlag, Überlingen-Bodensee.

HörREN, T. 2011: Bruchidius imbricornis (PANZER, 1795) - neu für die Rheinprovinz, nebst weiteren bemerkenswerten Arten aus einer Kiesgrube in Köln (Col., Bruchidae). - Mitteilungen der Arbeitsgemeinschaft Rheinischer Koleopterologen 21: 16-20.

Klausnitzer, B.; Hornig, U.; Behne, L.; Franke, R.; Gebert, J.; Hoffmann, W.; Jäger, O; Müller, H.; Richter, W.; Sieber, M. \& Vogel, J. 2018: Die Käferfauna (Coleoptera) der Oberlausitz. Teil 3: Nachträge, Gesamtübersicht und Analyse der Umweltbezüge. - Entomologische Nachrichten und Berichte, Beiheft 23, 632 S., 305 Abb., 1 Karte. 
Kühn, E.; Musche, M.; Harpke, A.; Feldmann, R.; Ulbrich, K.; Wiemers, M. \& Settele, J. 2019: Tagfalter-Monitoring Deutschland: Jahresauswertung 2018. - Oedippus 36: 6-38.

LoRenz, J. 1996: Oxythyrea funesta (PoDA) in Dresden gefunden. - Entomologische Nachrichten und Berichte 40: 185 .

Medger, G. 2019: Käferparadies an der Seseke. Naturreport 23: 38-40.

Medvedev, S. I. 1964: Plastinchatousye (Scarabaeidae), posdem. Cetoniidae, Valginae, Fauna SSSR, zhestkokrylye, tom 10, vyp. 5. - Moskva, Leningrad: Isdatel'stvo Akademii Nauk SSSR: 375 S. (in Russisch).

Micó, E. \& Galante, E. 2003: Biology and new larval descriptions for three Cetoniine beetles (Coleoptera: Scarabaeidae: Cetoniinae: Cetoniini: Cetoniina, Leucocelina). - Annals of the Entomological Society of America 96: 95-106. - doi: 10.1603/0013-8746(2003)096[0095:BANLDF]2.0 .CO;2.

MünCh, M.; Nuss, M. \& Seidel, J. 2010: Das Glühwürmchen (Lamprohiza splendidula (LinNaEus, 1767)) in Sachsen. - Zusammenfassung der Ergebnisse der sächsischen Suchaktion "Wo tanzt das Glühwürmchen?” aus den Jahren 2007-2009 (Coleptera: Lampyridae). - Sächsische Entomologische Zeitschrift 5: 40-48.

Neumann, V.; Süssmuth, T. \& Thurow, A. 2014: Der Trauer-Rosenkäfer Oxythyrea funesta (PoDA, 1761) (Coleoptera, Scarabaeidae, Blatthornkäfer) in Sachsen und Sachsen-Anhalt. - Entomologische Nachrichten und Berichte 58: 199-200.

Niehuis, M. \& Weitzel, M. 2009: Der Trauer-Rosenkäfer - Oxythyrea funesta (PodA, 1761) - in RheinlandPfalz und im Saarland (Coleoptera: Lamellicornia: Cetoniidae). - Fauna Flora Rheinland-Pfalz 11: 789-814.

Ødegand, F. \& Tømmeras, B. A. 2000: Compost heaps - refuges and stepping-stones for alien arthropod species in northern Europe. - Diversity and Distribution 6: 45-59. - doi: 10.1046/j.14724642.2000.00071.x.

Peter, M.; $\quad$ Diekötter, T. \& Kremer, K. 2019 : Participant outcomes of biodiversity citizen science projects: A systematic literature review. Sustainability 11: 2780. - doi: 10.3390/su11102780.
Pocock, M. J. O.; Chandler, M.; Bonney, R.; ThornHILl, I.; Albin, A.; $\quad$ August, T.; Bachman, S.; Brown, P. M. J.; Cunha, D. G. F.; Grez, A.; Jackson, C.; Peters, M.; Rabarijaon, N. R.; Roy, H. E.; Zaviezo, T. \& Danielsen, F. 2018: A vision for global biodiversity monitoring with citizen science. Advances in Ecological Research 59: 169-223. - doi: 10.1016/bs.aecr.2018.06.003.

Rössner, E. 2012: Die Hirschkäfer und Blatthornkäfer Ostdeutschlands (Coleoptera: Scarabaeoidea). - Verein der Freunde und Förderer des Naturkundemuseums Erfurt e.V., Erfurt: 508 S.

Schaffrath, U. 1997: Beitrag zur Kenntnis der Blatthornund Hirschkäfer (Col.: Trogidae, Geotrupidae, Scarabaeidae, Lucanidae) in Nordhessen. - Phillipia 8: $121-130$

Smetana, A. 2006: Unterfamilie Cetoniinae. Catalogue of Palaeartic Coleoptera (ed. I. Lobl \& A. SmetanA), Band 3: 283-313. - Apollo Books, Stenstrup, Denmark.

Subchev, M.; Toshova, T.; Andreev, R.; Petrova, V.; Maneva, V.; Spasova, T.; Marinova, N.; Minkov, P. \& VelcheV, D. 2012: Using floral baited colour traps for detection and seasonal monitoring of Oxythyrea funesta (PoDA) (Coleoptera: Cetoniidae) in Bulgaria. - Acta Zoologica Bulgarica 64: 439-443.

TAmutis, V. \& DApkus, D. 2013: Distribution of Oxythyrea funesta (PoDA, 1761) (Coleoptera: Scarabaeidae, Cetoninae) in Lithuania. - Zoology and Ecology 24: 33-39. - doi: 10.1080/ 21658005.2013.870366.

Thomaes, A.; Renneson, J.-L.; Drumont, A. \& DeschepPER, C. 2016: Range shift of Oxythyrea funesta PoDA, 1761 in Belgium (Coleoptera, Cetoniidae). Lambillionea 116: 187-195.

VON HoldT, E. 2012: Entomologische Besonderheiten der Jahre 2010 und 2011. - NABU Hannover Hannoverscher Vogelschutzverein von 1881 e.V. (HVV) 1/2012: 3-6.

Vuts, J.; Imrei, Z. \& Tóth, M. 2008: Development of an attractant-baited trap for Oxythyrea funesta PoDA (Coleoptera: Scarabaeidae, Cetoniinae). - Zeitschrift für Naturforschung 63: 761-768.

ZINkE, J. 1997: Oxythyrea funesta (PoDA). - Entomologische Nachrichten und Berichte 41: 212. 